\title{
Secuestro pulmonar extralobar asintomático y la importancia del diagnóstico y tratamiento perinatal: El manejo expectante no es una opción terapéutica
}

\author{
Rogelio Sancho-Hernández,* ${ }^{*}$ Lizbeth Solorio-Rodríguez,* Anné Azriel Durán-Colín,* \\ Yolanda Iribe-Gaxiola, ${ }^{*}$ Francisco Javier Cuevas-Schacht, * Eduardo López-Corella*
}

*Instituto Nacional de Pediatría, Ciudad de México;

Hospital Materno Perinatal Mónica Pretelini Sáenz, Ciudad de Toluca, Estado de México.

Trabajo recibido: 01-VI-2015; aceptado: 19-VIII-2015

\begin{abstract}
RESUMEN. Introducción: La ultrasonografía prenatal representa actualmente una forma de presentación de las malformaciones congénitas broncopulmonares. $90 \%$ se manifiesta en neonatos con obvias anormalidades radiológicas y con una evolución sintomática donde la resección quirúrgica está claramente indicada. En < 4\% ocasionan efecto de masa con desviación mediastinal, hidropesía y muerte fetal; y en otro extremo, se comportan «asintomáticas» con involución aparente y su manejo ha sido controversial: resección electiva y temprana o conducta expectante hasta documentar la regresión radiológica o el desarrollo de complicaciones. Se analiza la literatura en torno a la controversia existente: operar o no operar. Descripción de casos: Tres fetos con el diagnóstico prenatal de masa sólida intratorácica, neonatos asintomáticos: uno con hidrotórax y otro sin aparente involución prenatal, ambos con irrigación anómala compatibles con secuestro extralobar; el tercero con una masa paracardíaca donde los estudios de imagen no documentaron un secuestro extralobar de localización ectópica y sincrónica con malformación adenomatoidea quística, sólo los hallazgos transquirúrgicos y patológicos confirmaron el diagnóstico. Discusión: Las malformaciones congénitas broncopulmonares asintomáticas podrían ser diagnosticadas y resecadas quirúrgicamente por su historia natural no reconocida y por el bajo índice de regresión natural, las propuestas radiológicas no son eficaces para practicar la conducta expectante.
\end{abstract}

Palabras clave: Secuestro extralobar, resección electiva, malformación asintomática.

ABSTRACT. Introduction: Prenatal ultrasonography currently represents presentation form of bronchopulmonary malformations. To $90 \%$ manifest in neonates with obvious radiological abnormalities and symptomatic progression where surgical resection is clearly indicated. In < $4 \%$ causing mass effect with mediastinal shift, hydrops and fetal death and other extreme behave «asymptomatic» with apparent involution and its management has been controversial: early elective resection or radiologic expectant management to document the regression or development complications. It analyzes the literature about the controversy: operate or not to operate. Description of cases: Three fetuses with prenatal diagnosis of intrathoracic solid mass, asymptomatic newborns: one with hydrothorax and other without apparent prenatal involution both with abnormal irrigation compatible with extralobar sequestration, the third with a paracardiac mass where imaging studies did not diagnostic a extralobar sequestration of ectopic location and synchronous with cystic adenomatoid malformation, only transoperative and pathological findings confirmed the diagnosis. Discussion: In asymptomatic patients all bronchopulmonary malformations could be diagnosed and surgically resected for unrecognized their natural history and the low rate of natural regression, radiological proposals are not effective for monitoring.

Key words: Extralobar sequestration, elective resection, asymptomatic malformation.

\section{INTRODUCCIÓN}

El diagnóstico prenatal mediante la ultrasonografía obstétrica es una nueva forma de presentación de las malformaciones congénitas broncopulmonares; ${ }^{1}$ estas lesiones intratorácicas podrían ocasionar en la evolución intrauterina efecto de masa con desviación mediastinal, hidropesía, polihidramnios y muerte fetal; y en otro extremo, comportarse como lesiones que experimentan regresión o involución aparente, ignorándose la evolución e historia natural de dichos comportamientos perinatales. ${ }^{1,2}$ En una etapa posnatal se manifiestan en recién nacidos con obvias anormalidades radiológicas y una evolución sintomática con dificultad respiratoria, insuficiencia cardíaca e infección, donde la resección quirúrgica es claramente indicada. Sin embargo, algunos lactantes se comportan asintomáticos y su manejo ha sido controversial: un grupo de expertos aboga por una resección quirúrgica temprana y electiva, y otro grupo recomienda la intervención quirúrgica sólo después 
del desarrollo de síntomas o complicaciones y propone una conducta expectante con evaluaciones clínicas y radiológicas con tomografía computada o resonancia magnética hasta constatar la regresión. ${ }^{3}$

El grupo expectante aboga por la observación con base en que estas malformaciones son asintomáticas en su mayoría y representan hallazgos incidentales, la historia natural es desconocida y existe la posibilidad de regresión sin los riesgos y desventajas operatorios y anestésicos de la toracotomía y la resección quirúrgica. Por otro lado, el grupo quirúrgico argumenta que con la cirugía temprana y electiva se evitan las dificultades técnicas asociadas a la resección de un tejido infectado y fibrosado, estas lesiones «no representan una variante normal» y la posibilidad de permanecer asintomáticas es baja, existe un riesgo de malignidad asociada y la recuperación posoperatoria neonatal es rápida. ${ }^{3,4}$ Por lo anterior, se presentan tres casos representativos y se analiza la revisión de la literatura en torno a la controversia existente: operar o no operar.

\section{PRESENTACIÓN DE CASOS}

Caso 1. Feto de 30 semanas de gestación (SDG) con diagnóstico prenatal de masa intratorácica sólida y posterior recibió irrigación aberrante de la aorta torácica y cursó con derrame pleural, que al condicionar riesgo de hipoplasia pulmonar se le realizó toracocentesis única intrauterina del hemitórax izquierdo obteniéndose $20 \mathrm{~mL}$ de trasudado amarillo con polimorfonucleares en $80 \%$, cariotipo normal, cursando posteriormente sin hidropesía fetal y sin desviación mediastinal, sin placentomegalia y sin datos de preclampsia o síntomas maternos de alarma, cantidad de líquido amniótico normal. Se obtiene producto masculino de término y asintomático, en la evaluación radiológica posnatal por angiotomografía se documenta un vaso sistémico anómalo tributario de la aorta torácica irrigando la lesión sólida sin datos de involución ni regresión. A los 5 días de vida se decide por minitoracotomía izquierda la resección de una masa de apariencia lingular sólido-quística con pleura visceral propia, se realiza el control vascular con corte y sutura de su vaso aórtico torácico aberrante compatible con secuestro pulmonar extralobar, se retira el drenaje pleural a las $24 \mathrm{~h}$ del posquirúrgico y egresa con evolución satisfactoria (figura 1).

Caso 2. Feto de 23.1 SDG con una masa ecogénica en el lóbulo inferior del pulmón derecho recibiendo irrigación de un vaso nutricio aparentemente tributario de las ramas pulmonares de $24 \times 15 \times 31 \mathrm{~mm}$, sin datos de derrame pleural ni desviación mediastinal y sin morbilidad materna presente. En un control ultrasonográfico posterior a las 35.1 SDG persiste la masa ecogénica con un componente quístico agregado de $20 \times 15 \times 20 \mathrm{~mm}$ con aparente disminución de tamaño, pero persistiendo con la misma irrigación aberrante ya
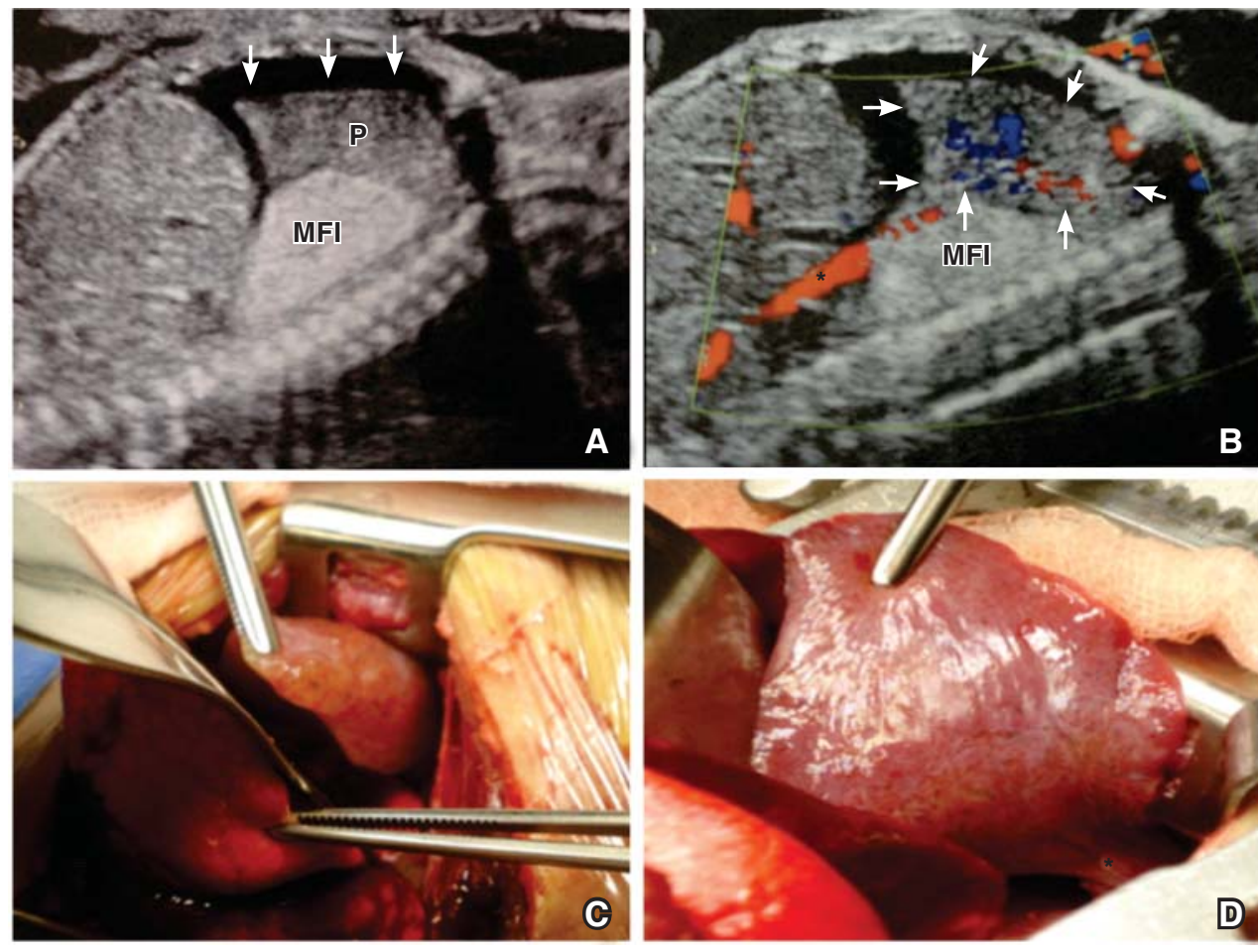

\section{Figura 1.}

A. Masa fetal intratorácica (MFI) sólida (microquística) en el diagnóstico prenatal a las 30 SDG, con derrame pleural (flechas) que requirió toracocentesis in utero al condicionar riesgo de hipoplasia pulmonar (P); en B. Pulmón irrigado por la circulación menor (flechas), se advierte un vaso aórtico sistémico hacia el interior de la MFI ( ${ }^{*}$ amarillo); C. Lesión piramidal quístico-sólida con pleura visceral propia en su cara anterior; D. Cara posterior sólida mostrando un vaso aórtico torácico aberrante (* amarillo) en su base compatible con secuestro pulmonar extralobar. Imagen a color en: www.medigraphic.com/ neumologia 
descrita compatible con secuestro pulmonar intralobar y probable malformación adenomatoidea quística, sin desplazamiento mediastinal y con una relación lesión/ circunferencia cefálica (CVR) de 0.1 traduciendo riesgo bajo de desarrollar hidropesía fetal, sin datos de morbilidad materna (figura 2).

Se obtiene neonato de término y asintomático, en la evaluación radiológica posnatal con tomografía se advierte una lesión sólida posterior de $45 \times 35 \times 10 \mathrm{~mm}$, triangular y basal del pulmón derecho sin componente quístico y sin irrigación anómala aparente, la evaluación ecocardiográfica expone una comunicación interauricular tipo foramen oval de $4.7 \mathrm{~mm}$ con cortocircuito de izquierda a derecha sin repercusión clínica evidente. Por la persistencia de la lesión con incremento en su tamaño y por la presencia de cortocircuito pulmonar con saturaciones de $90 \%$ que mejoraban con aporte de oxígeno hasta $94 \%$ se decidió realizar a los 3 días de vida minitoracotomía derecha encontrándose: lóbulo inferior derecho con zonas de congestión y atelectasia que experimenta mejoría posterior a la separación y liberación de una masa de apariencia piramidal de $4.0 \times 3.0 \times 0.7 \mathrm{~cm}$ sólido-quística con pleura visceral propia, se realiza el control vascular con corte y sutura de su vaso aórtico torácico aberrante incrementado la saturación a 98\% compatible con secuestro pulmonar extralobar con cortocircuito pulmonar, se retira el drenaje pleural a las $24 \mathrm{~h}$ del posquirúrgico y egresa con evolución satisfactoria (figura 3).

Caso 3. Feto de 28 semanas de gestación (SDG) con una masa paracardíaca izquierda sólida de $2 \mathrm{~cm}$ sin derrame pleural ni pericárdico, sin desviación mediastinal y sin vaso sistémico anómalo demostrable, no placentomegalia ni morbilidad materna agregada. Se obtiene neonato de término y asintomático, en la evaluación posnatal con radiología torácica, tomografía y resonancia magnética no se logra demostrar la masa intratorácica prenatal (figura 4). Por la persistencia de la masa en la evaluación estructural por ecocardiografía neonatal se decidió realizar a los 6 días de vida minitoracotomía diagnóstica encontrando una masa extrapericárdica de $2.5 \mathrm{~cm}$, delimitada por el diafragma, pericardio y esófago, de forma piramidal y cubierta por pleura mediastinal, de características sólido-quísticas recibiendo irrigación sistémica de dos vasos infradiafragmáticos y sin guardar comunicación con el esófago, la cual correspondió en el estudio histopatológico con un secuestro extralobar de localización atípica y sincrónica con malformación adenomatoidea quística tipo II, la evolución posquirúrgica fue satisfactoria y sin secuelas (figura 5).
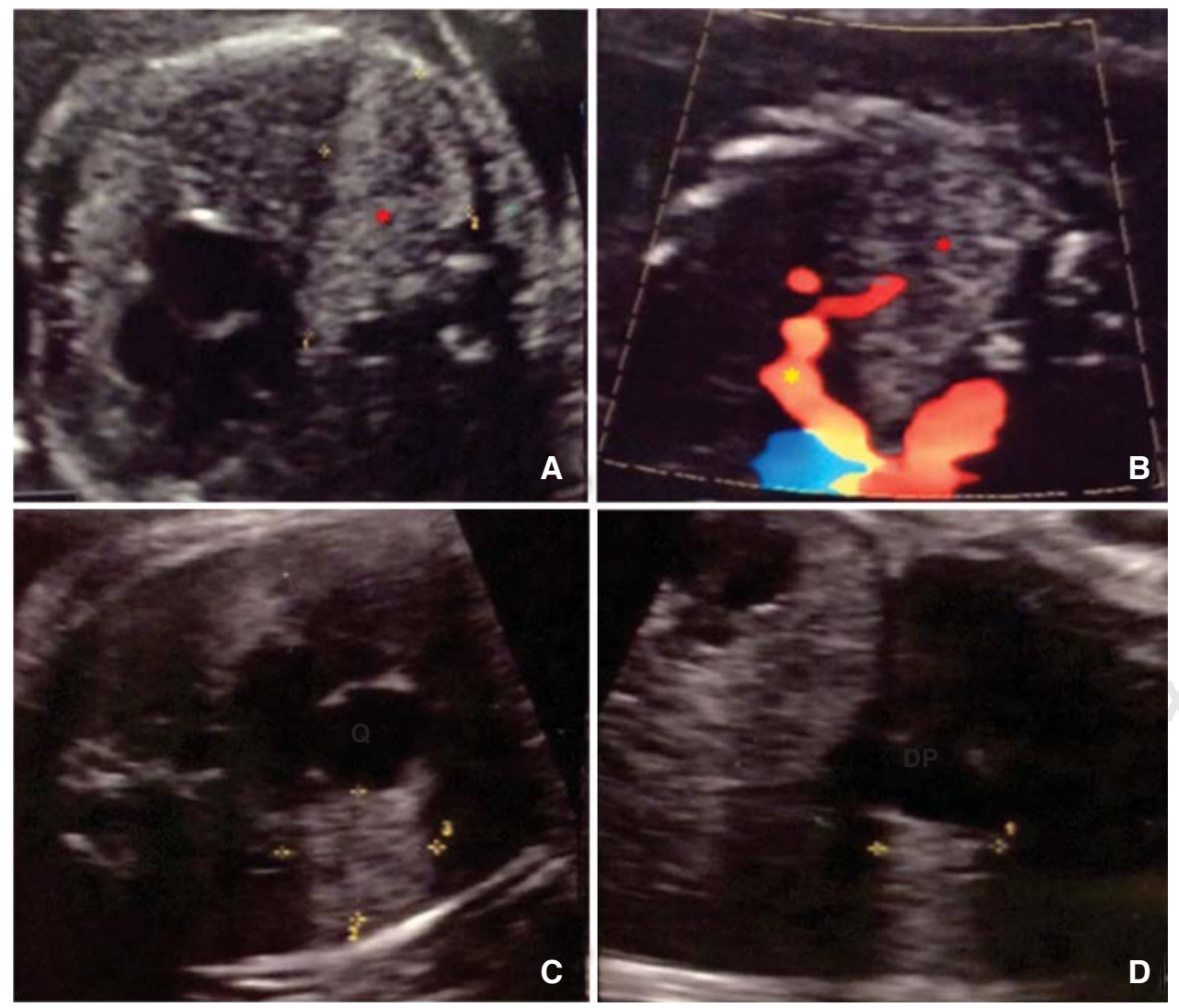

Figura 2.

A. Masa intratorácica fetal ecogénica-sólida ( ${ }^{*}$ rojo) de $24 \times 15 \times 31$ $\mathrm{mm}$ dependiente de lóbulo inferior derecho a las 23 SDG; en B. Vaso nutricio atípico ( ${ }^{*}$ amarillo) dependiente de ramas pulmonares sugerentes de un secuestro pulmonar intralobar o malformación adenomatoidea quística tipo III: en C. persistencia de la masa a las 35 SDG, aunque disminuyó de tamaño $(20 \times 21 \mathrm{~mm})$ se agrega un componente quístico $(Q)$; $\mathbf{D}$. lesión persistente con espesor de $20 \mathrm{~mm}$ con escaso derrame pleural (DP) que no condiciona desviación mediastinal ni riesgo de hidropesía fetal. Imagen a color en: www.medigraphic.com/ neumologia 

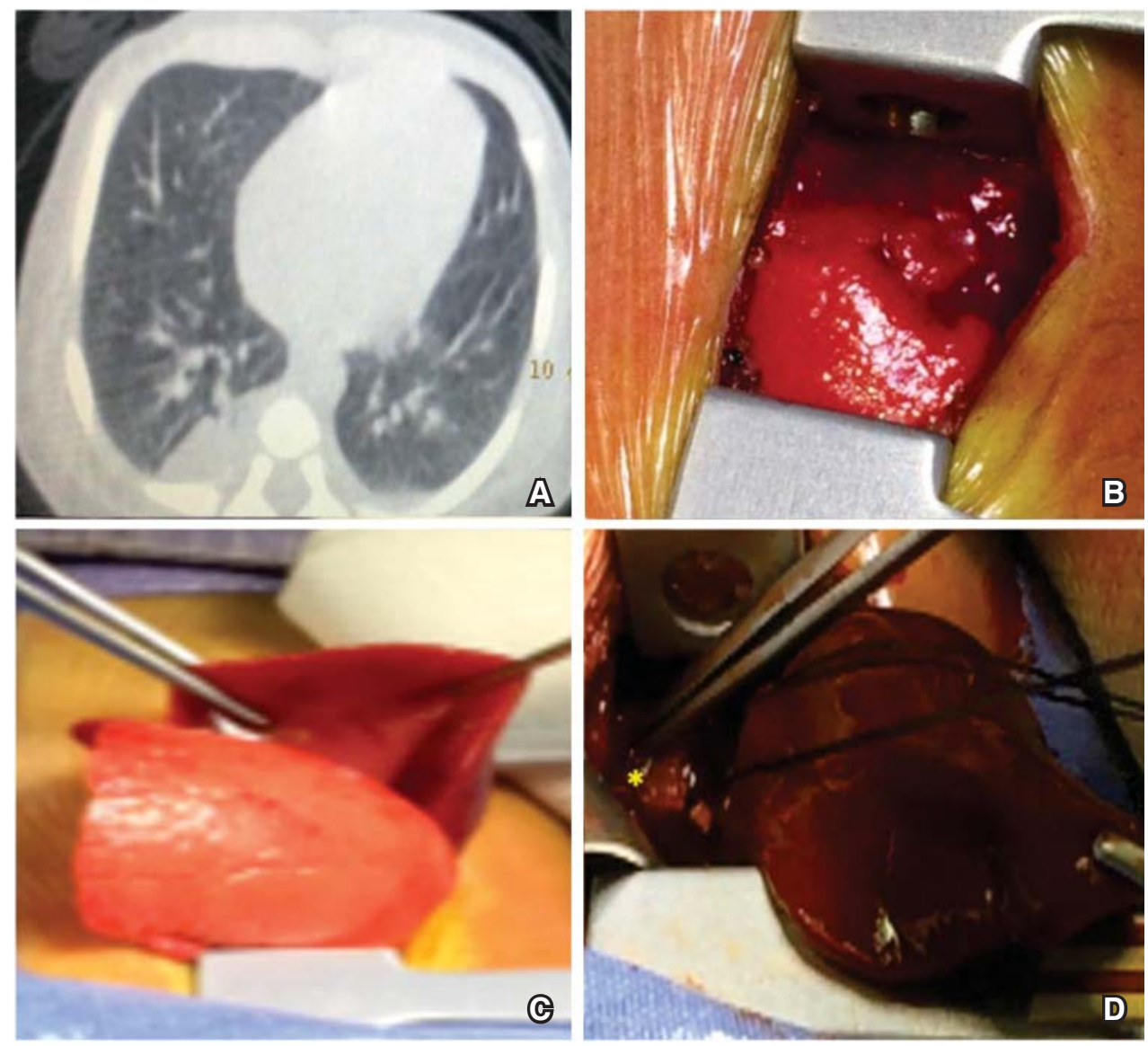

Figura 3.

A. Lesión sólida en tomografía posnatal de $45 \times 35 \times 10 \mathrm{~mm}$, delimitada en lóbulo inferior derecho y sin vaso sistémico demostrable; en B. Lóbulo inferior derecho congestivo con parches atelectásicos sugerentes de cortocircuito pulmonar; en C. Posterior al aislamiento de la lesión sólido-quística de $40 \times 30$ $\times 7 \mathrm{~mm}$, con pleura propia y $\sin$ ventilación (sujetado por pinzas) se aprecia perfusión y ventilación adecuadas para el lóbulo inferior adyacente; $\mathbf{D}$. Corte-sutura del vaso sistémico aberrante tributario de la aorta torácica ( ${ }^{*}$ amarillo) compatible con secuestro pulmonar extralobar.

Imagen a color en: www.medigraphic.com/ neumologia

\section{DISCUSIÓN}

La historia natural de las malformaciones congénitas broncopulmonares no está claramente descrita: desde lesiones sólidas prenatales con riesgo de hidrotórax, hidropesía y muerte fetal hasta la posibilidad de degeneración maligna, o bien la coexistencia con lesiones híbridas: malformación adenomatoidea quística (MAQ) y secuestro broncopulmonar (SBP) como en el presente caso, donde elaborar predictores del potencial de morbilidad resultan imposibles; ${ }^{5}$ más aún, el tamaño de las lesiones no guarda correlación clínica con aquellos pacientes que debutan con hidrotórax prenatal, infección, dificultad respiratoria o datos de cortocircuito sistémico pulmonar con insuficiencia cardíaca de alto gasto e hipertensión pulmonar. ${ }^{1,6}$ Se ha descrito la torsión e infarto de secuestro pulmonar extralobar (SPE) a nivel del pedículo aórtico aberrante que los irriga. ${ }^{7}$ Las lesiones congénitas quísticas pulmonares asintomáticas tienen el potencial de infectarse, de causar hemoptisis con hemotórax ${ }^{8,9}$ y degeneración maligna; ${ }^{3}$ el riesgo de desarrollar malignidad en estas lesiones ha sido reportado en más de 40 casos y $8.6 \%$ de neoplasias pulmonares malignas en la infancia fueron asociados a éstos (blas- toma pulmonar y carcinoma de células escamosas). ${ }^{10}$ Estas complicaciones severas de las malformaciones «asintomáticas» podrían ser evitadas por la resección temprana y electiva. En una revisión sistemática, las lesiones asintomáticas no conllevaron mortalidad con la resección quirúrgica, mientras que la morbilidad de $6.7 \%$ correspondió con fuga aérea y hemorragia, similar a los reportes previos para la resección quirúrgica en pacientes sintomáticos. ${ }^{11,12}$ Otro posible beneficio de la resección temprana es el crecimiento pulmonar compensatorio, incluso este crecimiento es más robusto en infantes que en adultos. Con el uso incrementado de la ultrasonografía prenatal, más lesiones quísticas congénitas pulmonares son diagnosticadas en el segundo y tercer trimestre de embarazo; no obstante, a pesar del avance tecnológico los errores diagnósticos son posibles: en una importante serie $8.6 \%$ de los casos tuvieron un diagnóstico posoperatorio diferente al diagnóstico radiológico prenatal y otro $8.6 \%$ tuvieron otros diagnósticos adicionales (quistes broncogénicos y neuroentéricos, lesiones híbridas)..$^{12}$ Estas lesiones híbridas diagnosticadas únicamente por histopatología sugieren la existencia de un origen común para las MAQ y los SBP. Parece ser que dentro de estas 

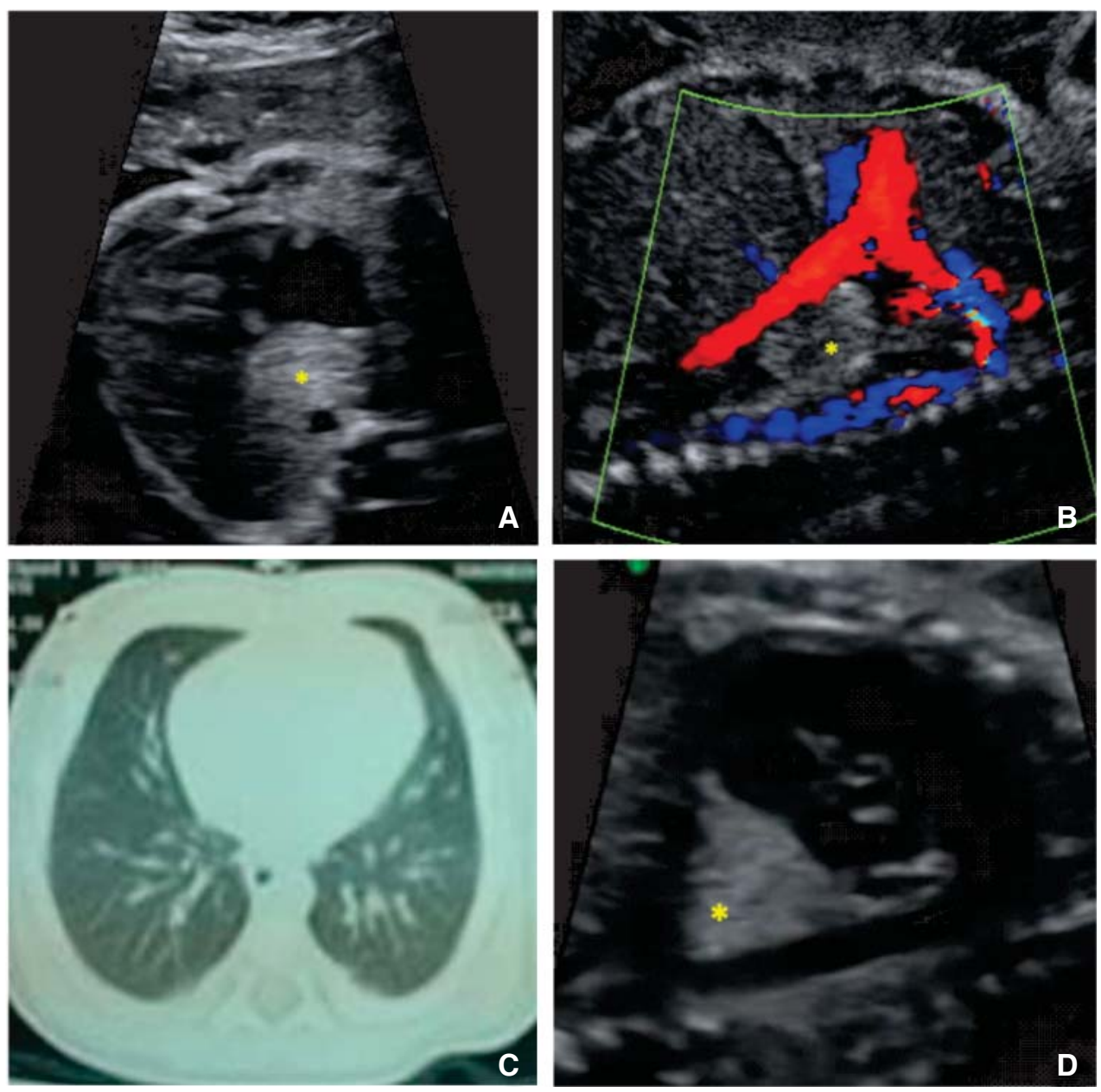

Figura 4.

A. Masa fetal paracardíaca (*amarillo): sólida, sin derrame pleural ni pericárdico, sin desviación mediastinal ni comorbilidad materna; en B. Lesión sin irrigación sistémico-pulmonar identificable; en C. Tomografía posnatal normal sin evidencia de la lesión paracardíaca izquierda; en D. persistencia de la lesión en la evaluación por ecocardiografía posnatal ( ${ }^{*}$ amarillo). Imagen a color en: www.medigraphic.com/ neumologia lesiones híbridas, la asociación de MAQ tipo 2 con SBP intralobar es la más frecuente, la asociación de MAQ tipo 2 con SPE con localización atípica y ectópica como en nuestro reporte, resulta extraordinaria y confunde las teorías de embriogénesis pulmonar. La MAQ usualmente ocurre en la etapa pseudoglandular mientras el SBP se desarrolla en la etapa embrionaria cuya irrigación es sostenida por la aorta dorsal, estos cambios dinámicos apoyan un origen común y podrían representar dos extremos de un amplio espectro patológico. ${ }^{13,14}$ Patológicamente estas lesiones muestran datos histológicos combinados de SBP intralobar y MAQ tipo II y III; $47 \%$ de estas lesiones fueron diagnosticadas prenatalmente, pero en ninguna se logró demostrar el aporte arterial sistémico, tal como ocurrió con nuestros reportes, al parecer la sobrevida para estas lesiones híbridas es favorable (94\%) cuando son resecadas por cirugía electiva, pero $33 \%$ de las lesiones inicialmente asintomáticas se comportaron sintomáticas con infección durante los primeros 4 meses de edad; sólo existen escasos 19 reportes que describen lesiones híbridas de SPE que contienen elementos histológicos de MAQ tipo $1 .{ }^{13}$ Por lo anterior, el diagnóstico prenatal no es un predictor confiable de las características histopatológicas de estas lesiones híbridas, la capacidad de regresión y la historia natural desconocida con localización ectópica y atípica como en nuestro reporte obliga siempre a diagnóstico diferencial transoperatorio e histopatológico confirmatorios en todos los pacientes asintomáticos y sintomáticos. Al parecer el SPE de localización intra o paracardíaca es explicado embriológicamente por la presencia de primordios pulmonares supernumerarios que podrían quedar incluidos dentro de los canales cardioperitoneales hacia la quinta semana de gestación en donde la irrigación de estos primordios se lleva a cabo por vasos sistémicos de la aorta dorsal. ${ }^{15} \mathrm{El}$ diagnóstico diferencial con MAQ, teratoma, hemangioma, fibroma, quistes pericárdicos y broncogénicos obliga a su confirmación histológica en todos los casos, como en nuestro reporte son sospechados cuando en la ultrasonografía prenatal se presentan como masas sólidas 

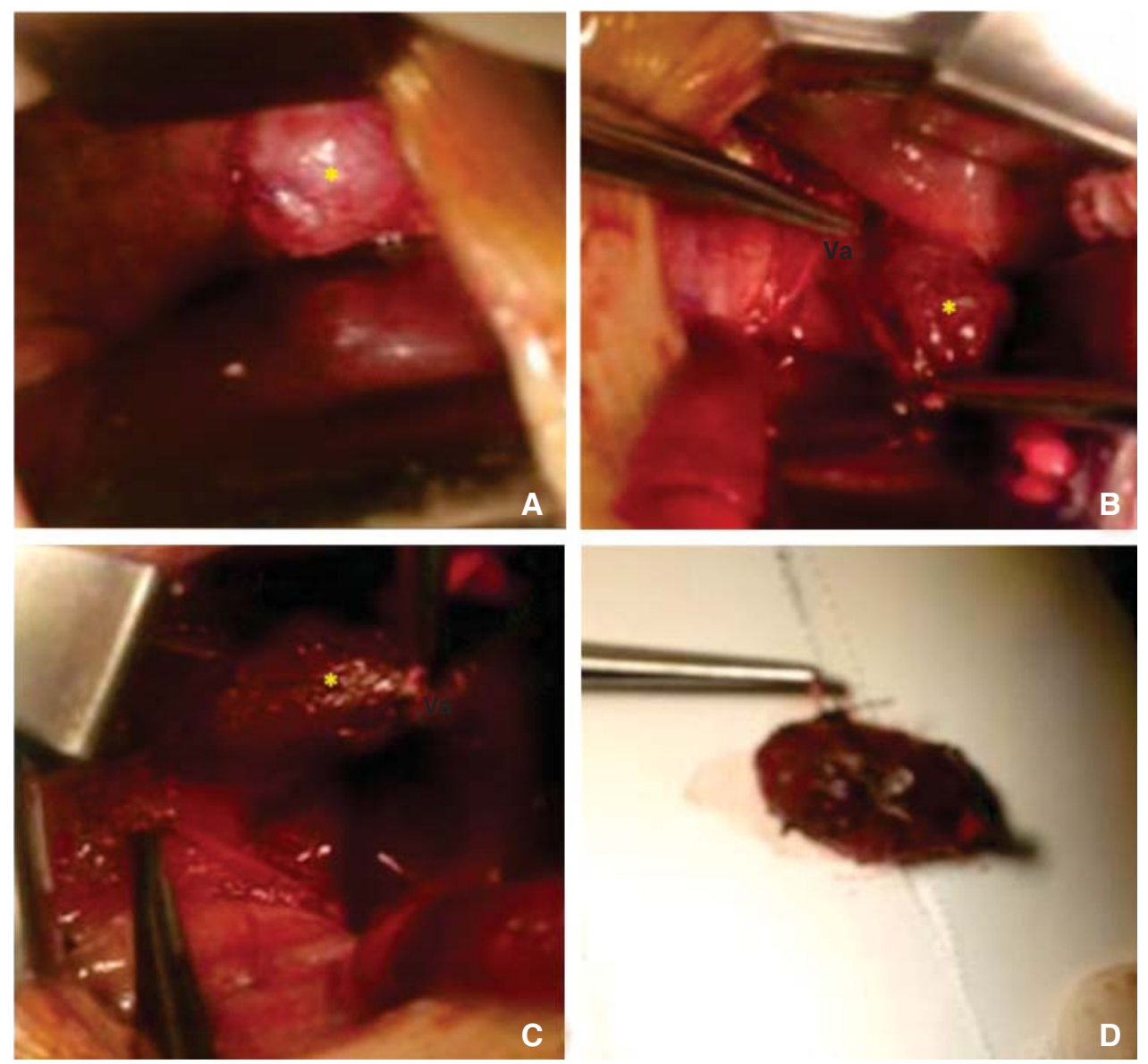

\section{Figura 5.}

A. Masa paracardíaca (* amariIlo) de $25 \mathrm{~mm}$, extrapericárdica y delimitada por el diafragma y esófago; en B. Lesión piramidal cubierta por pleura mediastinal, de características sólidoquísticas recibiendo irrigación sistémica de dos vasos infradiafragmáticos (va) no identificados por radiología; en C. Lesión $\left(^{*}\right)$ sin guardar comunicación con el esófago (pinza señalando nervio vago); D. Secuestro extralobar (pinza sostiene vaso sistémico aberrante) de localización atípica y sincrónica con malformación adenomatoidea quística tipo II en el examen patológico.

Imagen a color en: www.medigraphic.com/ neumologia paracardíacas o intrapericárdicas acompañados de hidropesía fetal, derrame pericárdico o efecto de masa; por su rareza sólo 5 casos han sido reportados en la literatura y fomentan la controversia de las teorías embrionarias de un origen común de las malformaciones broncopulmonares congénitas. ${ }^{15-18}$

Más aún, podríamos considerar que ante la historia natural desconocida de estas lesiones híbridas: la escasa posibilidad de involución, la morbilidad infecciosa y la degeneración maligna de la MAQ podrían acentuarse exponencialmente por una irrigación sistémica aberrante de estos SBP ectópicos y de localización atípica.

Estos diagnósticos divergentes o adicionales en torno a las lesiones congénitas broncopulmonares asintomáticas sustentadas en la sola vigilancia radiológica podrían resultar en obviar el diagnóstico y tratamiento quirúrgico oportunos de estas lesiones «híbridas» con riesgo exponencial de complicar, la falta de sensibilidad de las modalidades radiológicas para elaborar un diagnóstico diferencial de estas lesiones, así como la baja morbilidad asociada a la resección quirúrgica neonatal soporta el argumento para resecar estas malformaciones asintomáticas. ${ }^{12}$
Un metaanálisis de la literatura en torno a las malformaciones quísticas broncopulmonares de diagnóstico prenatal documenta que la regresión antenatal se presentó en 18\% y la involución sólo en 11\%; 17\% debuta de manera sintomática y requiere una intervención quirúrgica de emergencia neonatal y las complicaciones tempranas asociadas a esta conducta corresponden a $28 \%$ de los casos con fuga aérea, infección, hemorragia y derrame pleural, las complicaciones tardías se presentan en $10 \%$ y corresponden con enfermedad residual, infección, bronquiolitis, síndrome de dificultad respiratoria y asma. 7.5\% muere después de una intervención quirúrgica de emergencia. En la conducta de la cirugía electiva neonatal se presentan menos de $4.5 \%$ de complicaciones tempranas, ni complicaciones tardías ni muerte fueron documentadas con esta conducta. ${ }^{1}$ La edad promedio en la que los lactantes manifiestan sintomatología es entre los 7 y 10 meses (tiempo que coincide con la declinación de las inmunoglobulinas protectoras maternas) y este grupo sintomático expresa el mayor índice de complicaciones, concluyendo que la cirugía electiva está asociada a un mejor pronóstico que la cirugía emergente. ${ }^{1}$ Las técnicas quirúrgicas de 
mínima invasión por toracoscopia proveen una propuesta diagnóstica y terapéutica para la resección del SPE con disminución de la estancia intrahospitalaria y resultados cosméticos aceptables; sin embargo, el método de abordaje no parece influir en el índice de complicaciones. ${ }^{1,19}$

Una nueva alternativa para los SBP es la embolización del vaso anómalo por cateterismo, pero la experiencia acumulada al momento no ha mostrado resultados superiores o definitivos a la opción quirúrgica: algunas de estas lesiones deben permanecer en vigilancia por una regresión incompleta y también se han documentado focos sépticos y abscesos en lesiones residuales posteriores a la embolización. Por lo anterior, consideramos que la sola interrupción del aporte vascular de los SBP y de aquellas lesiones híbridas podría ser insuficiente en erradicar un tejido pulmonar residual embriológicamente anómalo y no funcional, existe una posibilidad de neovascularización de estas lesiones remanentes. ${ }^{19-21}$

Se ha propuesto a la tomografía con contraste como la modalidad diagnóstica preferida e inclusive el pilar de la vigilancia y manejo expectante en los pacientes asintomáticos; ${ }^{3}$ sin embargo, existen reportes similares a los nuestros, en los que la tomografía preoperatoria no ha identificado en número y disposición la totalidad de los múltiples vasos aberrantes que irrigan a los SPE, información crucial para la constante vigilancia y control vascular transoperatorios en los que varios casos de hemorragia severa y muerte han sido documentados en la resección no advertida de estos vasos, cambios histopatológicos muestran que estas arterias son elásticas y ateroescleróticas que pueden tornar a los vasos extremadamente retráctiles y friables. ${ }^{22-25}$ Consideramos que el diagnóstico de estas malformaciones sustentado en la radiología no es $100 \%$ eficaz más aún en aquellas lesiones de disposición atípica (infradiafragmáticas o paracardíacas) cuya evolución ultrasonográfica prenatal y búsqueda ecocardiográfica de malformaciones asociadas podrían ser otras consideraciones diagnósticas a la tomografía (TAC) y resonancia magnética (RM); en nuestro último caso estas modalidades de radiología no fueron capaces de advertir la lesión paracardíaca, ni la doble irrigación aberrante infradiafragmática o la existencia del componente quístico de la malformación adenomatoidea; existe además un reporte creciente de malformaciones broncopulmonares sincrónicas con posibilidad de transformación maligna como la malformación adenomatoidea expuesta en nuestro registro, donde hasta hoy, es difícil predecir en estas lesiones sincrónicas o híbridas cuáles podrían progresar al hidrotórax con hidropesía fetal, a la estabilidad en su tamaño o a la involución y regresión. Los SPE de diagnóstico prenatal son típicamente sólidos, como en nuestros casos, y la presencia de aire posnatal en ausencia de infección podría significar una comunicación esofágica, hallazgo extraordinario que obligaría entonces a la exploración quirúrgica para descartar quistes de duplicación digestiva o neuroentéricos. La incidencia de infección en los secuestros intralobares es de $91 \%$ contra $14 \%$ de los extralobares y se han reportado que estos SPE infectados tuvieron por hallazgo un bronquioesofágico. ${ }^{3,12}$ Por lo anterior, la resección de las malformaciones congénitas broncopulmonares asintomáticas incluido el secuestro pulmonar extralobar, es la terapéutica recomendada sustentada en las siguientes premisas:

a) La historia natural de los SPE que son diagnosticados como masas pulmonares prenatales es variable y exigen un diagnóstico diferencial histopatológico obligado con el neuroblastoma o la malformación adenomatoide quística tipo III, más aún si el aporte vascular aberrante no puede ser demostrado.

b) La posibilidad estadística de que las malformaciones broncopulmonares detectadas prenatalmente experimenten «regresión» $(18 \%)$ o «involución» (11\%) es muy baja; las lesiones híbridas o sincrónicas con un aporte vascular sistémico importante podrían tener nula oportunidad de involución.

c) Las lesiones «pequeñas» que experimentaron involución o disminución gradual de su tamaño prenatalmente podrían ser vigilados conservadoramente durante el período neonatal, fuera de este grupo etáreo el tamaño de la lesión no guarda correlación con la sintomatología ni la capacidad de regresión.

d) Al no existir indicadores pronósticos claros en la literatura actual sobre el potencial de malignidad e infección del SBP ni de las lesiones sincrónicas asociadas a éste, la conducta expectante con clínica y radiología permanece como una opción experimental.

e) La cirugía electiva neonatal está asociada a un mejor pronóstico sobre la morbimortalidad que la cirugía emergente. La cirugía electiva podría recomendarse hasta la lactancia temprana menor de 6 meses, más que por circunstancias estadísticas por el momento inmunológico favorable de este grupo de edad.

f) La resección quirúrgica temprana de lesiones asintomáticas entre los 3-6 meses de edad es una recomendación apoyada por los grandes centros materno-fetales y es imprescindible durante este período electivo cubrir las inmunizaciones contra gérmenes encapsulados que podrían complicar con infecciones que atenten contra la vida del paciente. 


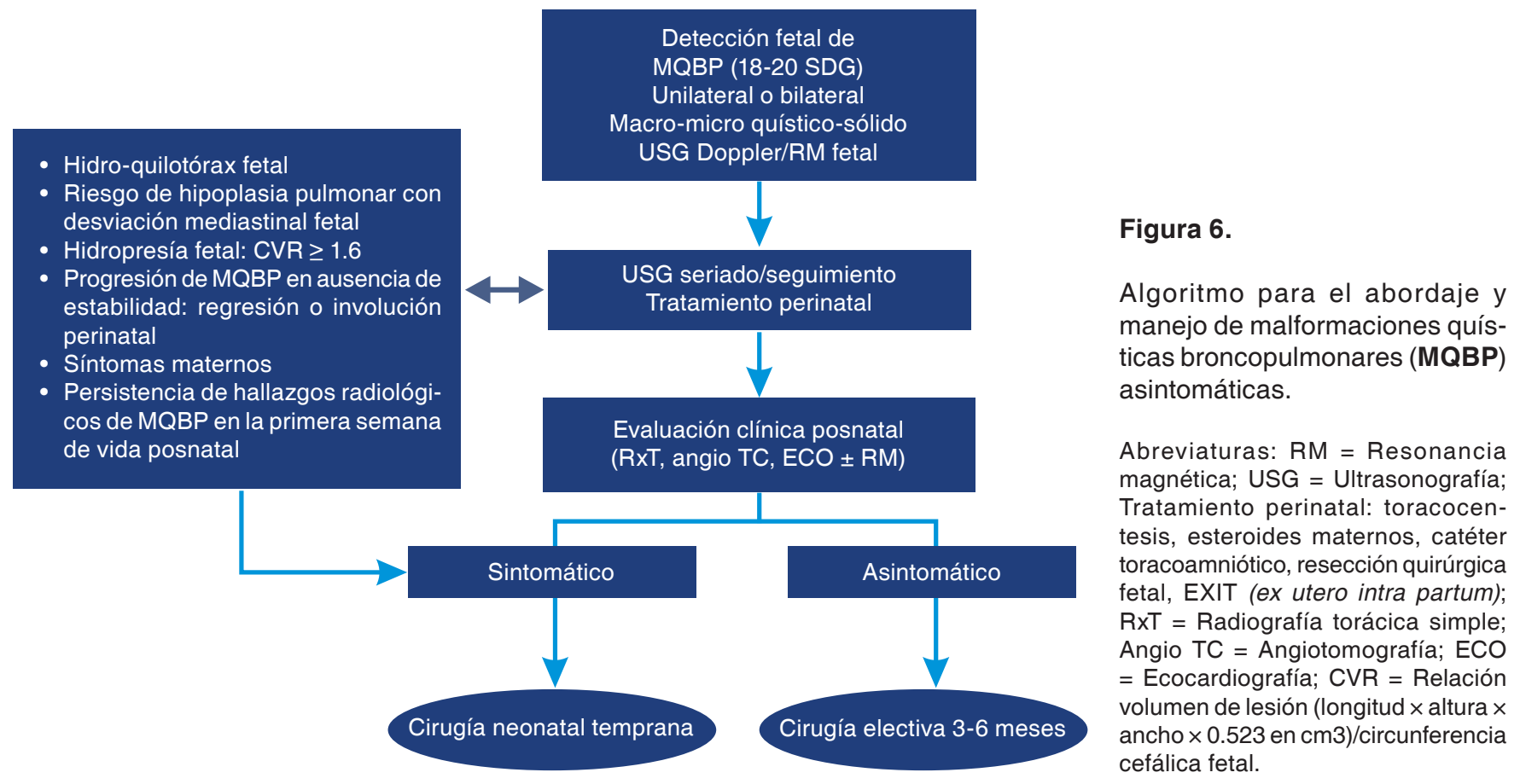

g) Los métodos radiológicos (TAC y RM) recomendados para la vigilancia expectante de las malformaciones broncopulmonares no son suficientemente integrales, proporcionarlos a nuestra población asintomática como método de vigilancia a mediano o largo plazo no es factible por factores sociodemográficos y culturales, se requiere un extenso período de seguimiento para poder recomendar el manejo conservador de las lesiones asintomáticas. ${ }^{26,27}$

Nuestra conducta perinatal propone que son manifestaciones prenatales «sintomáticas» las siguientes: a) la presencia de hidrotórax y quilotórax fetales con riesgo de adquirir hipoplasia pulmonar, b) la morbilidad materna (polihidramnios, preeclampsia), c) la desviación mediastinal y/o hidropesía fetal y d) la progresión de la lesión con ausencia para documentar estabilidad, regresión o involución perinatales (hasta los primeros siete días de vida). De tal forma que la presencia de estas manifestaciones prenatales complementados con la persistencia de hallazgos radiológicos posnatales inmediatos representan una expresión sintomática que requiere resección quirúrgica dentro del período neonatal y se propone un algoritmo de manejo que requiere ser validado en futuros estudios (figura 6).

Consideramos que aun en pacientes asintomáticos todas las malformaciones broncopulmonares que no muestran oportunidad de estabilidad en su crecimiento o involución perinatal (desde las 28 SDG hasta los primeros siete días de vida) podrían ser diagnosticados y resecados quirúrgicamente por su historia natural no reconocida y por el bajo índice de regresión natural, así como por las complicaciones asociadas a la infección y la potencial malignización en nuestra población con variadas limitantes socioculturales para proporcionar una conducta conservadora y expectante... «no existen variantes normales en torno a las malformaciones congénitas broncopulmonares y su diagnóstico diferencial obliga a la confirmación histopatológica».

\section{REFERENCIAS}

1. Stanton M, Njere I, Ade-Ajayi N, Patel S, Davenport M. Systematic review and meta-analysis of the postnatal management of congenital cystic lung lesions. J Pediatr Surg 2009;44(5):1027-1033. doi: 10.1016/j. jpedsurg.2008.10.118.

2. Parikh D, Samuel M. Congenital cystic lung lesions: is surgical resection essential? Pediatr Pulmonol 2005;40(6):533-537.

3. Laberge JM, Puligandla P, Flageole H. Asymptomatic congenital lung malformations. Semin Pediatr Surg 2005;1481):16-33.

4. Laberge JM, Bratu I, Flageole $H$. The management of asymptomatic congenital lung malformations. Paediatr Respir Rev 2004;5 Suppl A:S305-S312.

5. Adzick NS. Management of fetal lung lesions. Clin Perinatol 2009;36(2):363-376. x. doi: 10.1016/j. clp.2009.03.001. 
6. Aziz D, Langer JC, Tuuha SE, Ryan G, Ein SH, Kim PC. Perinatally diagnosed asymptomatic congenital cystic adenomatoid malformation: to resect or not? J Pediatr Surg 2004;39(3):329-334.

7. Huang EY, Monforte HL, Shaul DB. Extralobar pulmonary sequestration presenting with torsion. Pediatr Surg Int 2004;20(3):218-220.

8. Rubin EM, Garcia H, Horowitz MD, Guerra JJ Jr. Fatal massive hemoptysis secondary to intralobar sequestration. Chest 1994;106(3):954-955.

9. Avishai V, Dolev E, Weissberg D, Zajdel L, Priel IE. Extralobar sequestration presenting as massive hemothorax. Chest 1996;109(3):843-845.

10. Hancock BJ, Di Lorenzo M, Youssef S, Yazbeck S, Marcotte JE, Collin PP. Childhood primary pulmonary neoplasms. J Pediatr Surg 1993;28(9):1133-1136.

11. Al-Bassam A, Al-Rabeeah A, Al-Nassar S, et al. Congenital cystic disease of the lung in infants and children (experience with 57 cases). Eur J Pediatr Surg 1999;9(6):364-368.

12. Tsai AY, Liechty KW, Hedrick HL, et al. Outcomes after postnatal resection of prenatally diagnosed asymptomatic cystic lung lesions. J Pediatr Surg 2008;43(3): 513-517. doi: 10.1016/j.jpedsurg.2007.10.032.

13. Cass DL, Crombleholme TM, Howell LJ, Stafford PW, Ruchelli ED, Adzick NS. Cystic lung lesions with systemic arterial blood supply: a hybrid of congenital cystic adenomatoid malformation and bronchopulmonary sequestration. J Pediatr Surg 1997;32(7):986-990.

14. Chen HW, Hsu WM, Lu FL, et al. Management of congenital cystic adenomatoid malformation and bronchopulmonary sequestration in newborns. Pediatr Neonatol 2010;51(3):172-177. doi: 10.1016/S1875-9572(10)60032-0.

15. Yildiz K, Ozcan N, Cebi M, Köse N, Karakaya F. Intrapericardial extralobar pulmonary sequestration: unusual cause of hydrops fetalis. J Ultrasound Med 2005;24(3):391-393.

16. Benatar A, Vaughan J, Nicolini U, Trotter S, Corrin B, Lincoln C. Prenatal pericardiocentesis: its role in the management of intrapericardial teratoma. Obstet Gynecol 1992;79(5 (Pt 2)):856-859.

17. Todros T, Gaglioti P, Presbitero P. Management of a fetus with intrapericardial teratoma diagnosed in utero. J Ultrasound Med 1991;10(5):287-290.

18. Sydorak RM, Kelly T, Feldstein VA, et al. Prenatal resection of a fetal pericardial teratoma. Fetal Diagn Ther 2002;17(5):281-285.
19. Cho MJ, Kim DY, Kim SC, Kim KS, Kim EA, Lee BS. Embolization versus surgical resection of pulmonary sequestration: clinical experiences with a thoracoscopic approach. J Pediatr Surg 2012;47:2228-2233. doi: 10.1016/j.jpedsurg.2012.09.013.

20. Yeh CN, Wang JN, Tsai YC, Yao CT, Lin CS, Wu JM. Coil embolization of pulmonary sequestration in two infants: a safe alternative management. Acta Paediatr Taiwan 2006;47(2):88-91.

21. Lee KH, Sung KB, Yoon HK, et al. Transcatheter arterial embolization of pulmonary sequestration in neonates: long term follow up results. J Vasc Interv Radiol 2003;14(3):363-367.

22. Brown EG, Marr C, Farmer DL. Extralobar pulmonary sequestration: The importance of intraoperative vigilance. J Ped Surg Case Reports 2013;1(4):74-76. doi:10.1016/j. epsc.2013.02.015

23. Savic B, Birtel FJ, Tholen W, Funke HD, Knoche R. Lung sequestration: report of seven cases and review of 540 published cases. Thorax 1979;34(1):96-101.

24. Osaki T, Kodate M, Takagishi T, Nomi M, Murakami J, Yamamoto $\mathrm{H}$. Unique extralobar sequestration with atypical location and aberrant vessels. Ann Thorac Surg 2010:90(5);1711-1712. doi: 10.1016/j.athoracsur.2010.04.031.

25. Samuel M, Burge DM. Management of antenatally diagnosed pulmonary sequestration associated with congenital cystic adenomatoid malformation. Thorax 1999;54(8):701-706.

26. Di Prima FA, Bellia A, Inclimona G, Grasso F, Teresa M, Cassaro MN. Antenatally diagnosed congenital cystic adenomatoid malformations (CCAM): Research review. J Prenat Med 2012;6(2):22-30.

27. Singh R, Davenport M. The argument for operative approach to asymptomatic lung lesions. Semin Pediatr Surg 2015;24(4):187-195. doi: 10.1053/j.sempedsurg.2015.02.003.

\section{$\triangle$ Correspondencia:}

Rogelio Sancho-Hernández

Instituto Nacional de Pediatría. Insurgentes Sur Núm. 3700-C, Colonia Insurgentes Cuicuilco, 04530, Del. Coyoacán, México, D.F.

Cel. 044555252999

E-mail: saherog@yahoo.com.mx 